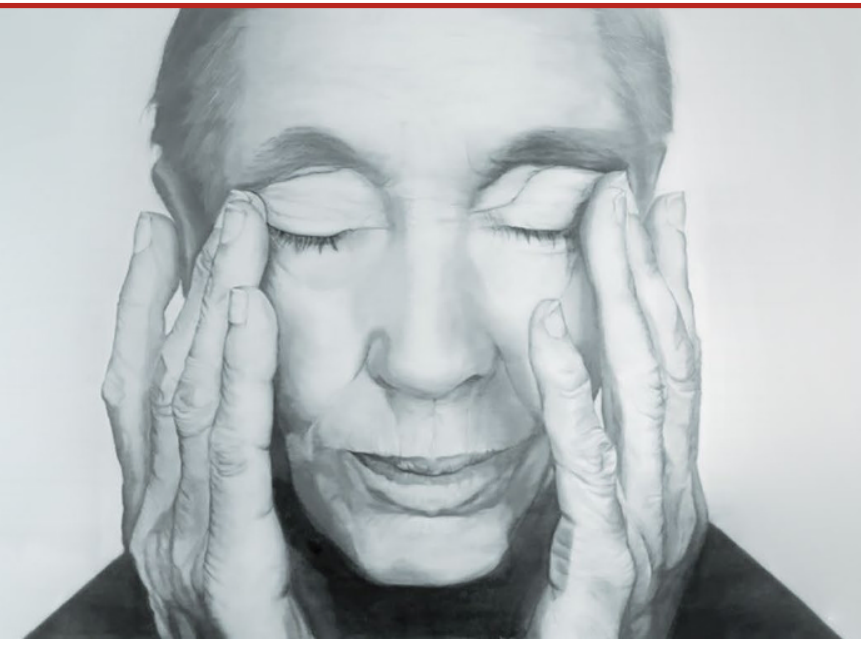

Thomas Ots

Graz, Österreich

\title{
Der gestresste oder sich stressende Mensch?
}

Ich eile durch die Innenstadt. Eine Patientin sieht mich: „Grüß Gott, Herr Doktor. Im Stress?", und schaut etwas mitleidsvoll. „Wieso im Stress? Ich gehe nur schnell. Habe viel zu erledigen. " Jetzt schaut sie so, als ob sie mir nicht ganz glaubt. Aber sie sagt es nicht.

Seit Jahren stehe ich im Kampf mit einem falschen Verständnis von Stress, wie er sich landauf, landab eingenistet hat: „Jaja, der Stress macht mich krank." In diesem Verständnis ist der Stress etwas von außen auf das Individuum Einwirkendes. Nein, der Stress ist nur bedingt etwas Äußerliches. Stress - so wie es auch Hans Selye definierte - entsteht in uns als die Summe von Adaptationsvorgängen und Reaktionen körperlicher wie psychischer Art, also als Antwort auf bestimmte (soziale) äußere Bedingungen oder Einwirkungen - oder eben nicht [1]. Es hängt also von unserer Reaktion auf diese ab. Habe ich ausreichend Fähigkeiten erworben, mit äußeren Stressoren umzugehen? Oder fehlen mir diese? Wenn in meiner Ordination ein Patient vom Stress spricht, der ihn krank macht, dann antworte ich mit einem Stan-

Deutsche Zeitschrift für Akupunktur 2019 • 62 (1): 21-23

https://doi.org/10.1007/s42212-019-0146-z

Online publiziert: 12. Februar 2019

○ Springer Medizin Verlag GmbH, ein Teil von

Springer Nature 2019 dardszenario: „Stellen Sie sich vor, in diesem Raum sitzen drei Patienten. Auf einmal geht die Tür auf und der Kopf von Tyrannosaurus Rex schaut herein: Ein Patient fürchtet sich und macht sich fast in die Hose, ein anderer bekommt ob dieser Überraschung einen Lachanfall, der letzte ärgert sich über diesen vermeintlichen, blöden Ulk von mir. Sehen Sie, Tyrannosaurus ist nicht der Stress, es hängt davon ab, wie sie reagieren. Also müssen wir schauen, a) was Sie für Stress in Ihrem Leben halten und b) wie Sie damit besser umgehen können“ (Betonung auf „müssen wir schauen“).

Ein großer Teil meiner Arbeit als psychosomatisch orientierter Arzt besteht darin,

a. dass die Patienten gemeinsam mit mir die Bedingungen und Lebensumstände, beruflichen Situationen etc. herausfinden, die sie „stressen“. Das ist die Vorbedingung. Dann geht es daran,

b. die eigentliche Entstehung des Stresses im Ich zu vermitteln und auf diese Einsicht aufbauend

c. bessere Bewältigungsmechanismen erkennbar, lernbar und einüben zu lassen (siehe hierzu auch [2]).

Klingt nach Psychotherapie? Ist es nicht ganz, eher nur begleitend. Aber ein gesundes $\mathrm{Maß}$ an Empathie unsererseits und Adhärenz (gemeinsam) sind gefordert1 (Abb. 1).

\section{Akupunktur ist Leibtherapie}

Akupunktur hat zwei Wirkungen - sie ist Körpertherapie und Körperpsychotherapie. Einmal die faktische, biologisch-neurophysiologische Wirkung. Die ist teils segmental, teils über neuronale, muskuläre, humorale etc. Verknüpfungen organisiert. Will ich das Herz beeinflussen, steche ich dorsal Bl 15. Das entspricht dem Segment Th 5, dazu ventral KG 14 oder einen Locus etwas höher und links davon sowie einen Punkt auf dem Herzmeridian (vorzugsweise $\mathrm{He} 7$ ), vielleicht noch Pe 6 und am Ohr Punkt 100 in der Mitte der Concha inferior. Diese Kombination garantiert die faktische Reizung des Herzens. Der Rest ist dann die körpereigene Autoregulation.

Die zweite Wirkung könnte man die universelle Wirkung der Akupunktur nennen. Und die hat sehr viel mit dem Faktor Zeit zu tun, die verloren geht, wenn man wie einst Felix Mann die Nadel sticht und nur kurz verweilen lässt [3]. Der Faktor Zeit beginnt schon beim Erstgespräch. Jeder Mensch ist anders, besitzt eine eigene Mischung aus Genen, Kultur, Erziehung, sozialem Stand, Vorerfahrungen, Emotionalität, Coping Strategien und Ressourcen ... (Abb. 1c, d) Cave: Nicht jeder äußere Stressor ist gleich: Erfahrungen von Hunger, Kampf, Verletzung, Vergewaltigung und Flucht bilden einen qualitativ anderen Stressor als der durch von sei- 


\section{Was ist Stress?}

Das Objekt-orientierte oder auch

das kausaflineare Stress-Modell

\section{Was ist Stress?}

Der passive Patient

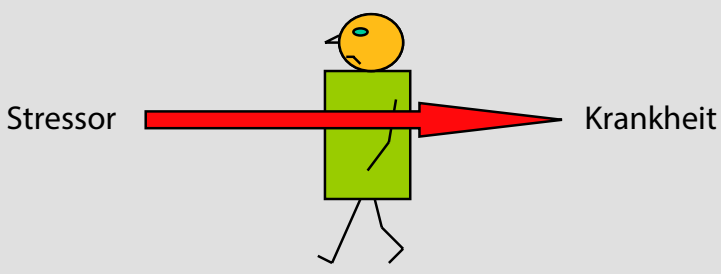

a

"Der Stress macht mich krank"

(Das Subjekt Mensch spielt faktisch keine Rolle)

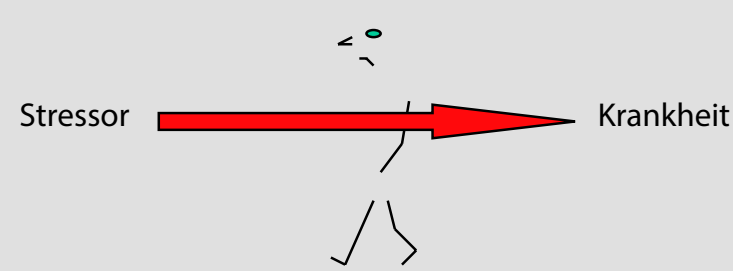

b

tet dieser Patient vom Arzt? "Bitte geben Sie mir etwas gegen Stress"

\section{Was ist Stress?}

Das Subjekt-orientierte Stressmodell

(z.B. Transaktionales Stressmodell vonLazarus)

\section{Was ist Stress?}

\section{Der Mensch als Subjekt}

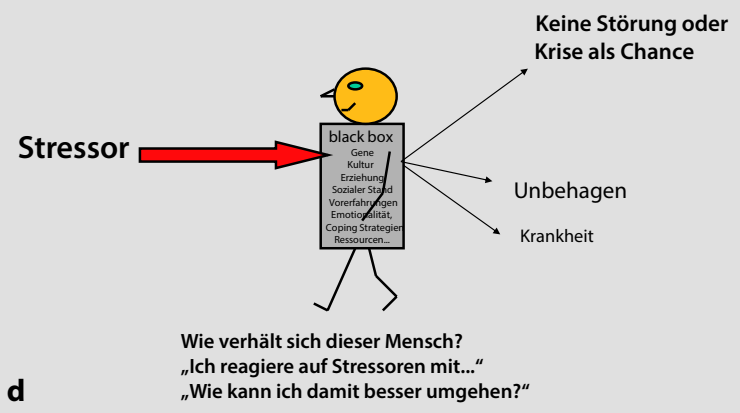

Abb. 1 Was ist Stress? a Das objektorientierte Stressmodell mit dem passiven Patienten (b). c Das subjektorientierte Stressmodell mit dem Menschen als agierendes Wesen (d)

nem Smartphone mit den asozialen Netzwerken und durch Energydrinks gehetzte neuzivilisatorisch geschädigte Mensch (um nur zwei Beispiele zu nennen). In manchen Fällen sind kaum noch individuelle Möglichkeiten von Stressverarbeitung gegeben. Davon mehr in anderen Artikeln dieses Praxisschwerpunkts.

Dann im Liegen oder ruhigen Sitzen während der Akupunkturtherapie beginnt der Patient sich wahrzunehmen. Zunächst rein körperlich als Objekt, als einen Körper habend. Vielleicht spürt er die aufliegenden Fersen, die Waden, den Hintern, die Schultern, den Hinterkopf. Dann hört und/ oder spürt er seinen Bauch - die Darmgeräusche und Darmbewegungen. Und plötzlich bemerkt er, dass er atmet. „Wie atme ich? Mein Gott, wie verkrampft oben im Brustkorb!“ Oder: „Was lastet auf mei- ner Brust?" Auf diese Wahrnehmung folgt oft ein tiefer Atemzug mit einer stoßweisen schnellen Ausatmung. Und gleich noch einmal. Und es ist möglich, dass mit der Ausatmung etwas als belastend Empfundenes ausgestoßen wird. Das tut gut. Und aus diesem Konglomerat von Empfindungen, Gedanken, aufkeimenden Erinnerungen entsteht leibliche Wahrnehmung. Leib verstanden als der beseelte (besser: bedeutsame) Körper. Das Liegen unter Nadeln in Ruhe und Zeit macht es möglich, dass unser Lehrmeister - die Atmung - in uns wirken kann. Es kommt zur - wie es der bekannte Körperpsychotherapeut und Mitglied der DZA-Redaktion, Helmut Milz, genial nannte: Wiederbeleibung [4]. Sich als Leib und nicht distanziert als Körper zu fühlen, ist somit ein Baustein der Wiedergewinnung des Ich als mir selbst wichtige Person. Und das benötigt Zeit und Ruhe als Grundlage innerer Achtsamkeit.

\section{Krise und Chance - Flasche halb leer oder halb voll?}

Ein Missverständnis geistert durch die Welt: Im Chinesischen gäbe es ein Wort, das sowohl Krise wie auch Chance bedeutet. Die des Chinesischen nicht mächtigen Menschen, und das sind im Westen die meisten, wissen nicht, dass der gemeinte chinesische Begriff „weiji“ aus zwei Wörtern (zwei Schriftzeichen) besteht:

- 危 „wei“ bedeutet Krise, Gefahr,

- 机 ,ji“ bedeutet sowohl Maschine, Mechanismus wie auch Dreh- oder Wendepunkt und letztlich Chance, Möglichkeit (viele chinesische Begriffe haben eine für uns nicht nachvollziehbare Vielbedeutung). 
Der zusammengesetzte Begriff 危机 „weiji“ wird in China aber nur als Krise verstanden, da in diesem Kontext des zusammengesetzten Begriffes als Krisenmechanismus gemeint.

Viele chinesische Begrifflichkeiten setzen sich aus zwei Zeichen zusammen. Im Idealfall meinen beide dasselbe oder Ähnliches. So wird die Bedeutung klarer:

- 会 „hui“ bedeutet: treffen, können, Chance,

- 机会 „jihui“ bedeutet somit: Chance.

Krise und Chance zusammen in einer Begrifflichkeit müsste also „weijihui“危机 会 heißen, dieser Begriff existiert aber im Chinesischen nicht.

Wenn man aber im psychosozialen Bereich arbeitet und Menschen eben diesen trostreichen/hoffnungsvollen Gedanken vermitteln möchte, könnte man eventuell etwas ungenau sein (der Zweck heiligt die Mittel) und sagen:

„Im Chinesischen gibt es einen Begriff (,weiji'), dessen einer Teil auch auf Chance hinweist, die sich hieraus ergibt.“

Und die Chance kann auch darin gesehen werden, dass die Flasche eben nicht halb leer, sondern halb voll ist. Es geht also darum, worauf wir unseren Fokus lenken, wie wir unsere Patienten unterstützen. Vom ehemaligen Vorsitzenden der DÄGfA, Wolfram Stör, stammt ein Ansatz, der die Patienten zunächst verwundert, dann - eventuell gar lachend - an die eigenen Kräfte glauben lässt: „Wie schaffst Du es, bei den guten Kräften, die Du hast, krank zu bleiben?“ [5].

\section{Resilienz, Empowerment und Sinn}

Resilienz (von lateinisch „resilire“: „zurückspringen“ „abprallen“) oder psychische Widerstandsfähigkeit ist die Fähigkeit, Krisen zu bewältigen und sie durch Rückgriff auf persönliche und sozial vermittelte Ressourcen als Anlass für Entwicklungen zu nutzen. Mit Resilienz verwandt sind Entstehung von Gesundheit (Salutogenese), Widerstandsfähigkeit (Hardiness), Bewältigungsstrategie (Coping) und Selbsterhaltung (Autopoiesis).

Das Gegenteil von Resilienz ist Verwundbarkeit (Vulnerabilität) ... [6].

Diese so kurze wie klare Wikipedia-Definition soll genügen. Resilient wird derjenige, der in der Flasche das sieht, was noch drin ist. Und das geht nur mit einem
Willen, mit einem Ziel, aus der Misere herauszukommen.

Hier greift auch der Begriff „Empowerment", der originär der US-amerikanischen Gemeindepsychologie entstammte.

Mit Empowerment (von englisch „empowerment“: „Ermächtigung, Übertragung von Verantwortung") bezeichnet man Strategien und Maßnahmen, die den Grad an Autonomie und Selbstbestimmung im Leben von Menschen oder Gemeinschaften erhöhen sollen und es ihnen ermöglichen, ihre Interessen (wieder) eigenmächtig, selbstverantwortlich und selbstbestimmt zu vertreten. Empowerment bezeichnet dabei sowohl den Prozess der Selbstbemächtigung als auch die professionelle Unterstützung der Menschen, ihr Gefühl der Macht- und Einflusslosigkeit („powerlessness“) zu überwinden und ihre Gestaltungsspielräume und Ressourcen wahrzunehmen und zu nutzen ... [7].

Und was, wenn alles gegen mich spricht und sich keine helfenden Ressourcen auftun?

Dann hilft es, an sich zu glauben, dem eigenen Leben einen Sinn zu geben, und wenn der Sinn einfach nur darin besteht, die aktuelle Krise zu meistern. Wir denken da an Viktor E. Frankl, dem Begründer der Logotherapie, der durch diesen Sinn das Konzentrationslager überlebte. Vor allem bei traumatisierten Menschen besteht die ärztliche Aufgabe darin, ihnen zu helfen, „Ja zum Leben zu sagen“ [8].

\section{Die Artikel dieses Praxisschwer- punkts}

geben ein beredtes Beispiel dafür, wie wir mit Körper- oder Ohrakupunktur, und hier im Besonderen mit dem NADAProtokoll (Raben, Schönegger, Friedrichs, Hase), mit dem Lösen körperlich-emotionaler Spannungen (Malek, Deim), mit verschiedenen traumafokussierten Psychotherapien (Barton, Röbe, Hase), mit nonverbaler Kommunikation (Raben), durch Ernährung und chinesische Arzneitherapie (Knauer), meist aber durch eine Kombination dieser Methoden ,gestressten " und traumatisierten, gar schwer traumatisierten Menschen helfen können, bis hin zu den neuen Randgruppen unserer Gesellschaft, den zu uns aus Hunger, Unterdrückung oder Krieg Geflüchteten (Friedrichs), die zumeist von Empower- ment im klassischen, sozialen Sinne nur träumen können.

Wie sich Fäden aus unterschiedlichen Richtungen verknüpfen

Es war nicht geplant, aber ein großer Teil dieser Ausgabe betrifft im weitesten Sinne das Thema „Psyche“. Hier sei auf den Grundlagenartikel von Sroka zur Bedeutung der Stimulation des Parasympathikus mittels Akupunktur hingewiesen, der deutlich in dieselbe Richtung zeigt: spannungslösend und regenerierend. Der Kommentar von Tauber-Bachmann beschäftigt sich mit dem Problem der Psyche, des Burn-outs und der Resilienz bei Ärzten. Auch die Rezensionen der Bücher von Cechura und Schache reihen sich in dieses Thema ein. Und um den Reigen komplett zu machen, führt die ÄfA (Ärzte für Akupunktur) Ende März in Wien ihren Kongress zu verschiedenen Wegen aus dem Stress durch: „Stress lass nach!“ Dort werden einige der Autoren dieses Schwerpunkts vortragen bzw. Kurse halten (Deim, Malek, Knauer, Ots).

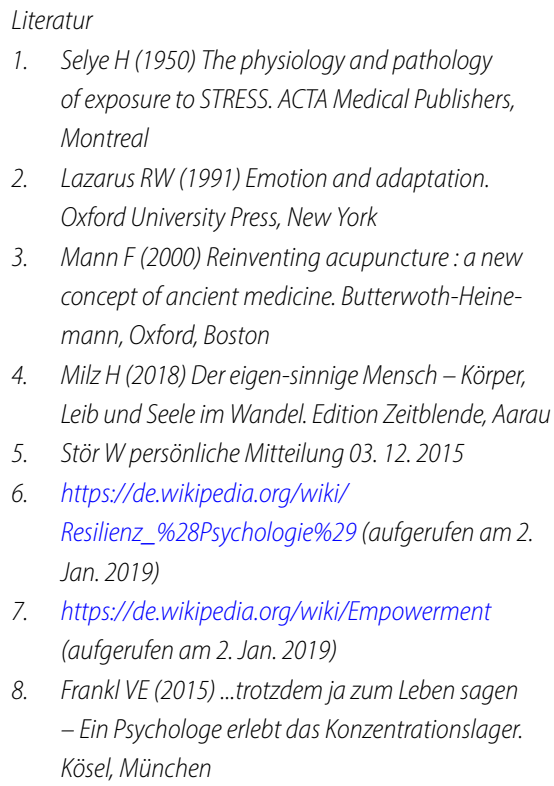

8. FrankIVE (2015)...trotzdem ja zum Leben sagen - Ein Psychologe erlebt das Konzentrationslager. Kösel, München

\section{Korrespondenzadresse}

\section{Thomas Ots}

Moserwaldweg 11

A-8042 Graz, Österreich

ots@daegfa.de

Interessenkonflikt. T. Ots gibt an, dass kein Interessenkonflikt besteht. 